\title{
Gamma-ray burst contributions to constraining the evolution of dark energy
}

\author{
S. Qi ${ }^{1,4}$, F.-Y. Wang ${ }^{2}$, and T. $\mathrm{Lu}^{3,4}$ \\ 1 Department of Physics, Nanjing University, Nanjing 210093, PR China \\ e-mail: qishi11@gmail.com \\ 2 Department of Astronomy, Nanjing University, Nanjing 210093, PR China \\ e-mail: fayinwang@nju.edu.cn \\ 3 Purple Mountain Observatory, Chinese Academy of Sciences, Nanjing 210008, PR China \\ e-mail: t.lu@pmo.ac.cn \\ 4 Joint Center for Particle, Nuclear Physics and Cosmology, Nanjing University - Purple Mountain Observatory, Nanjing 210093, \\ PR China
}

Received 11 April 2008 / Accepted 23 May 2008

ABSTRACT

\begin{abstract}
Aims. We explore the gamma-ray bursts' (GRBs') contributions in constraining the dark energy equation of state (EOS) at high $(1.8<z<7)$ and at middle redshifts $(0.5<z<1.8)$ and estimate how many GRBs are needed to get substantial constraints at high redshifts.

Methods. We estimate the constraints with mock GRBs and mock type Ia supernovae (SNe Ia) for comparisons. When constraining the dark energy EOS in a certain redshift range, we allow the dark energy EOS parameter to vary only in that redshift bin and fix EOS parameters elsewhere to -1 .

Results. We find that it is difficult to constrain the dark energy EOS beyond the redshifts of SNe Ia with GRBs unless some new luminosity relations for GRBs with smaller scatters are discovered. However, at middle redshifts, GRBs have comparable contributions with SNe Ia in constraining the dark energy EOS.
\end{abstract}

Key words. cosmological parameters - Gamma rays: bursts

\section{Introduction}

Since the discovery of the accelerating expansion of the universe (Riess et al. 1998; Perlmutter et al. 1999), a lot of work has been done on constraining the behavior of dark energy. In contrast to simple parametric forms for dark energy equation of state (EOS), such as $w(z)=w_{0}+w^{\prime} z$ (Cooray \& Huterer 1999) and $w(z)=w_{0}+w_{a} z /(1+z)$ (Chevallier \& Polarski 2001; Linder 2003), a nearly model-independent approach was introduced by Huterer \& Cooray (2005) to estimate the evolution of dark energy independently, and was adopted in analyses using type Ia supernova (SN Ia) data (Riess et al. 2007; Sullivan et al. 2007a,b). Qi et al. (2008) extended the approach to gamma-ray burst (GRB) luminosity data (Schaefer 2007), for which higher redshifts are accessible compared to SNe Ia. Though more stringent results have been obtained by this, the dark energy EOS at high redshifts, where we only have GRBs, is still totally unconstrained, except that it is most likely negative. This is primarily due to matter dominating dark energy at high redshifts and to dark energy becoming less important for determining the cosmic expansion; therefore, constraining it becomes more difficult. In this work we explore both how many GRBs are needed to get substantial constraints on dark energy EOS at high redshifts beyond those of SNe Ia and where the GRBs' contributions lie for a foreseeable number of GRBs.

\section{Methodology}

For the uncorrelated estimates of dark energy EOS, we first separate the redshifts into several bins and assume a constant dark energy EOS for each bin. Then we generate a Markov chain through the Monte-Carlo algorithm according to the likelihood function. The covariance matrix of the dark energy EOS parameters is calculated based on the Markov chain, and a transformation derived from the covariance matrix to decorrelate the EOS parameters. The evolution of dark energy is finally estimated out of the uncorrelated EOS parameters (see Huterer \& Cooray 2005, for details).

The process of generating Markov chains in these procedures is very time-consuming, especially when there are considerable observational/mock standard candles. We see that we need a large number of GRBs to get substantial constraints on dark energy EOS at high redshifts. Instead of using the uncorrelated approach directly, we adopt an alternative way to estimate this number. When estimating the constraints imposed on dark energy EOS in a certain redshift range, we allow the EOS parameter to vary only in that redshift bin and fix the EOS parameters elsewhere, say, to -1 . This is compatible with the uncorrelated approach to an approximate estimate, because the uncorrelated EOS parameters defined in Huterer \& Cooray (2005) are themselves localized well in redshift bins. Of course, the fixation of EOS at some redshifts will cause underestimates of the errors on the EOS at other redshifts, so the number we have estimated here can be viewed as a lower limit.

We determine the likelihood for EOS parameters from the $\chi^{2}$ statistic,

$\chi_{n}^{2}=\sum_{i=1}^{n} \frac{\left[\mu_{\mathrm{t}, i}(\theta)-\mu_{\mathrm{o}, i}\right]^{2}}{\sigma_{i}^{2}}$ 


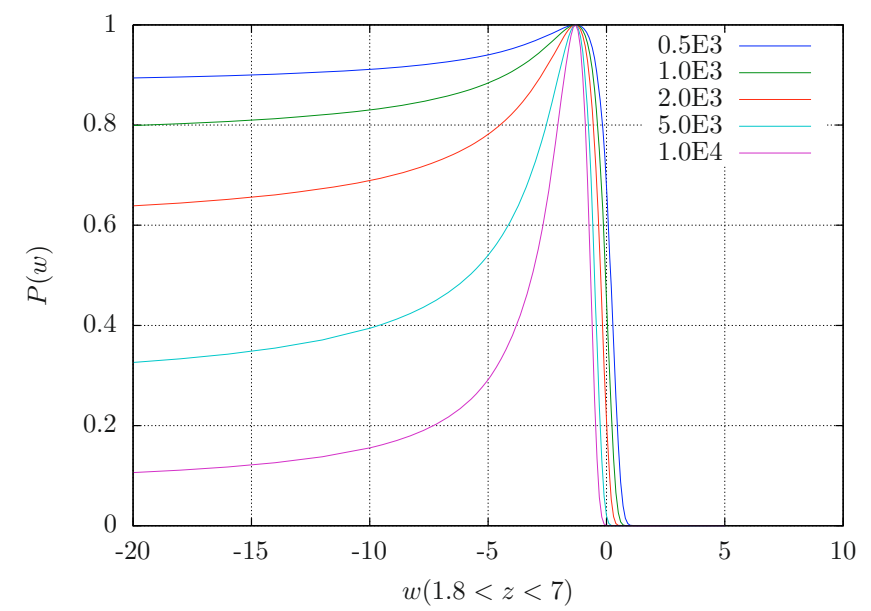

Fig. 1. Constraints from GRBs uniformly distributed in the redshift bin $1.8<z<7$ on the dark energy EOS parameter $w(1.8<z<7)$. The different lines stand for different number of GRBs. The probabilities are normalized to be 1 at the maxima.

where $\mu_{\mathrm{o}}$ is the observational/mock distance modulus of the standard candles with standard deviation $\sigma, \mu_{\mathrm{t}}(\theta)$ is the theoretically predicted distance modulus based on a cosmological model with parameter set $\theta$, and $n$ is the number of the standard candles. We constrain dark energy EOS parameters with mock data, whose generation involves random numbers (see the details below). To reduce the impact from the fluctuation of the mock data themselves, we generate many more standard candles than needed. For example, if we want to see the constraints imposed by $n$ samples of standard candles, we actually generate $N$ mock samples with $N \gg n$ and calculate $\chi_{N}^{2}$. Then $\chi_{n}^{2}$ is given by

$\chi_{n}^{2}=\frac{n}{N} \chi_{N}^{2}$

Throughout this paper, we choose $N=1.0 \mathrm{E} 5$.

The fiducial cosmological model we used to generate mock data is the flat $\Lambda$ CDM model with $\Omega_{m}=0.279$ and $H_{0}=$ $70.1 \mathrm{~km} \mathrm{~s}^{-1} \mathrm{Mpc}$ (see Komatsu et al. 2008). For SNe Ia, we approximate the intrinsic noise in distance moduli as a Gaussian scatter with a dispersion of $0.1 \mathrm{mag}$ and the observational errors as $0.23 \mathrm{mag}$, which are approximately the averages of the observational errors of the presently available SNe Ia (Riess et al. 2007; Wood-Vasey et al. 2007; Davis et al. 2007). The total error in our generated distance moduli for SN Ia is therefore $\sqrt{0.1^{2}+0.23^{2}}$ mag. We assume an uniform distribution for $\mathrm{SNe}$ Ia along the redshifts. For GRBs, instead of generating mock data about the five luminosity relations (see Schaefer 2007), we directly generate distance moduli like we do for SNe Ia for simplicity. The intrinsic scatter is set to be $0.65 \mathrm{mag}$, which is approximately the average of the errors of the GRBs' average distance moduli presented in Schaefer (2007), and we ignore the measurement uncertainties, which are less than the intrinsic scatter. We consider two kinds of distributions for GRBs in the redshift bin $1.8<z<7$. One is the uniform distribution, the other a very rough approximation to the distribution presented by Fig. 2 in Bromm \& Loeb (2006), i.e. $P(z) \propto \exp (-z / 7)$. We will see that our results are independent of the GRB distributions.

\section{Results}

Figures 1 and 2 show our results for the constraints from GRBs distributed in the redshift bin $1.8<z<7$ on the dark energy

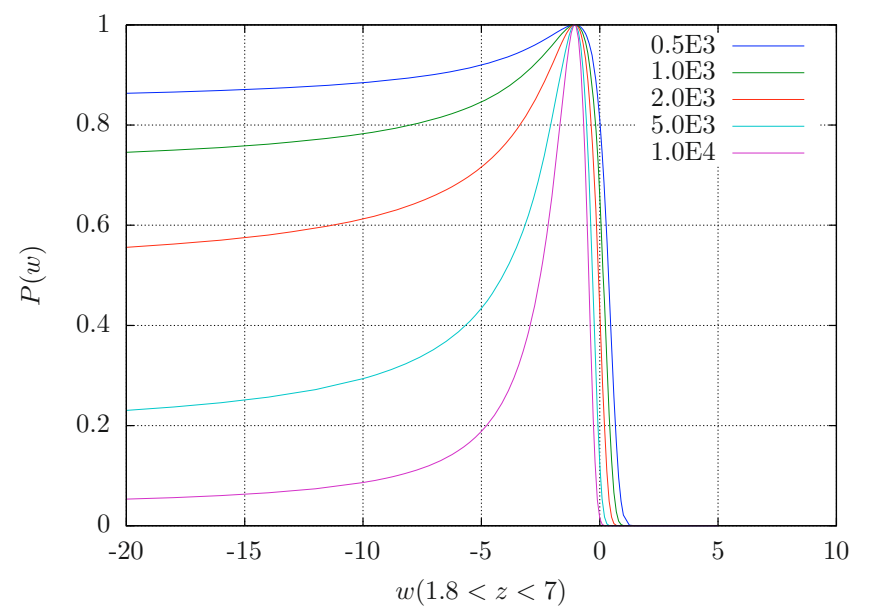

Fig. 2. Constraints from GRBs distributed in the redshift bin $1.8<z<$ 7 according to $P(z) \propto \exp (-z / 7)$ on the dark energy EOS parameter $w(1.8<z<7)$.

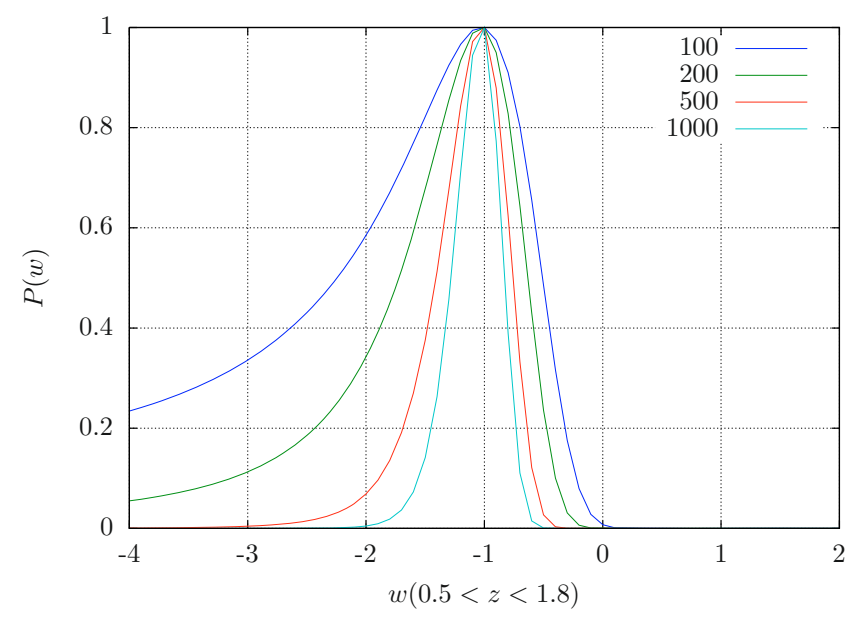

Fig. 3. Constraints from GRBs uniformly distributed in the redshift bin $1.8<z<7$ on the dark energy EOS parameter $w(0.5<z<1.8)$.

EOS parameter $w(1.8<z<7)$. We can see that, for a few hundred GRBs, the constraints are only the steep drop at about zero that is seen in the probability function of the EOS parameter $P(w)$. This is consistent with the results in Qi et al. (2008). Only when we have more than about 5000 GRBs can we begin to get concrete constraints on the EOS parameter. The GRBs' distributions have little impact on the conclusion; i.e., it is difficult to constrain the dark energy EOS parameters beyond the redshifts of SNe Ia with GRBs unless some new luminosity relations for GRBs with smaller scatters are discovered.

However, this does not mean that high-redshift GRBs contribute little to constraining the dark energy EOS parameters. It has been demonstrated in Qi et al. (2008) that, even with the presently available 69 GRBs (Schaefer 2007), the constraints could be improved significantly at redshifts $0.5 \lesssim z \lesssim 1.8$. Part of the improvement stems from GRBs beyond redshift 1.8. Because the luminosity distances of standard candles depend on the behavior of the dark energy through an integration over the redshift, high-redshift GRBs put constraints on dark energy at lower redshifts, where dark energy is important in determining the cosmic expansion. And since there are few GRBs at low redshifts, the contributions from GRBs would lie primarily in the middle redshifts. In Figs. 3 and 4 we explicitly show the constraints 


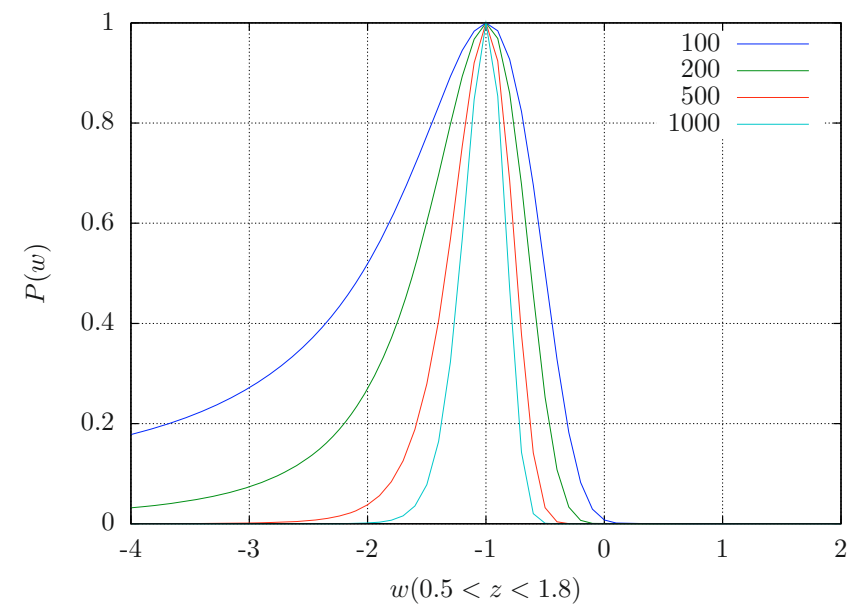

Fig. 4. Constraints from GRBs distributed in the redshift bin $1.8<z<7$ according to $P(z) \propto \exp (-z / 7)$ on the dark energy EOS parameter $w(0.5<z<1.8)$.

from GRBs distributed in the redshift bin $1.8<z<7$ on the dark energy EOS parameter $w(0.5<z<1.8)$. For comparison, we also plot the constraints from SNe Ia uniformly distributed in the redshift bin $0.5<z<1.8$ on the dark energy EOS parameter $w(0.5<z<1.8)$ in Fig. 5. We can see that the contributions from GRBs are comparable to that from $\mathrm{SNe}$ Ia.

\section{Summary}

We explored the GRBs' contributions in constraining the dark energy EOS at high redshifts $(1.8<z<7)$ and at middle redshifts $(0.5<z<1.8)$. When constraining the dark energy EOS in a certain redshift range, we allow the dark energy EOS parameter to vary only in that redshift bin and fix EOS parameters elsewhere to -1 . We find that it is difficult to constrain the dark energy EOS parameters beyond the redshifts of SNe Ia with GRBs unless some new luminosity relations for GRBs with smaller scatters are discovered. However, at middle redshifts, GRBs have contributions comparable with SNe Ia in constraining the dark energy EOS.

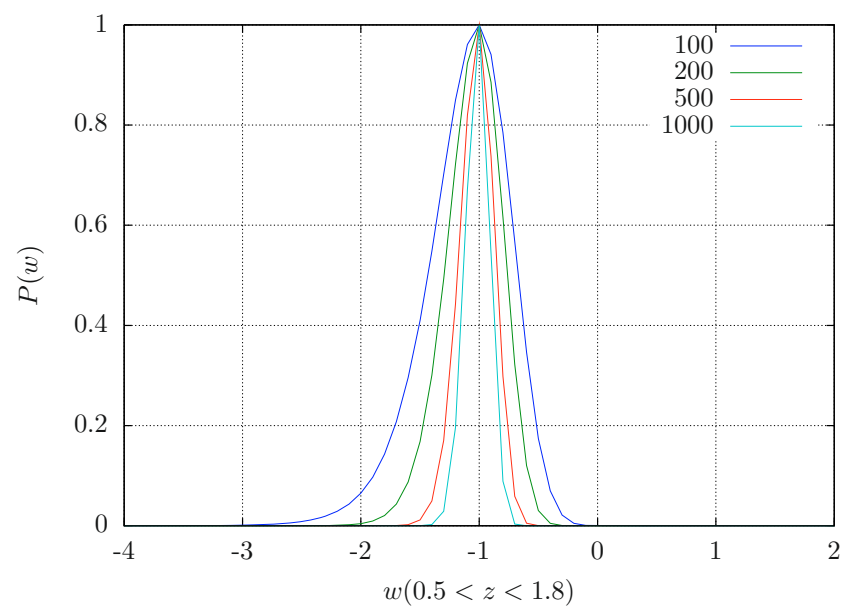

Fig. 5. Constraints from SNe Ia uniformly distributed in the redshift bin $0.5<z<1.8$ on the dark energy EOS parameter $w(0.5<z<1.8)$.

Acknowledgements. This work was supported by the Scientific Research Foundation of the Graduate School of Nanjing University (for Shi Qi), the Jiangsu Project Innovation for PhD Candidates CX07B-039z (for Fa-Yin Wang), and the National Natural Science Foundation of China under Grant No. 10473023.

\section{References}

Bromm, V., \& Loeb, A. 2006, AJ, 642, 382

Chevallier, M., \& Polarski, D. 2001, Int. J. Mod. Phys. D, 10, 213

Cooray, A. R., \& Huterer, D. 1999, AJ, 513, L95

Davis, T. M., Mörtsell, E., Sollerman, J., et al. 2007, AJ, 666, 716

Huterer, D., \& Cooray, A. 2005, Phys. Rev. D, 71, 023506

Komatsu, E., et al. 2008, [arXiv:0803.0547]

Linder, E. V. 2003, Phys. Rev. Lett., 90, 091301

Perlmutter, S., Aldering, G., Goldhaber, G. et al. 1999, AJ, 517, 565

Qi, S., Wang, F.-Y., \& Lu, T. 2008, A\&A, 483, 49

Riess, A. G., Filippenko, A. V., Challis, P., et al. 1998, AJ, 116, 1009

Riess, A. G., Strolger, L.-G.; Casertano, S., et al. 2007, AJ, 659, 98

Schaefer, B. E. 2007, AJ, 660, 16

Sullivan, S., Cooray, A., \& Holz, D. E. 2007a, JCAP, 0709, 004

Sullivan, S., et al. 2007b [arXiv:0709.1150]

Wood-Vasey, W. M., Miknaitis, G., Stubbs, C. W., et al. 2007, AJ, 666, 694 